\title{
Vida y milagros de Orsini Bertani \\ (Una semblanza: quehaceres de la cultura letrada del Novecientos)
}

\author{
Vida e milagres de Orsini Bertani \\ (Um perfil: tarefas da cultura letrada no início do século XX) \\ Life and miracles of Orsini Bertani \\ (A profile: chores of literacy culture at the beginning of the 20th century) \\ Pablo Rocca \\ Universidad de la República, Montevideo, Uruguay \\ $\diamond$
}

Resumen: La existencia de los impresos depende de la vida y la obra de libreros, impresores y editores. En las primeras tres décadas del siglo XX, en Montevideo, gracias a una combinación de varios factores, algunos de esos agentes contribuyeron a afirmar una cultura letrada débil y tambaleante. Entre ellos, figura crucial fue el italiano Orsini Bertani (1869-1939), que de activo anarquista en su país de origen, en Francia, Inglaterra y Argentina se volvió sosegado, fraterno y decisivo empresario cultural en la capital uruguaya. Interrogarse sobre sus días y quehaceres ayuda a comprenderlo y comprender la cultura de su época.

Palabras clave: Vida de Orsini Bertani; Impresos y actividad cultural; Uruguay (1900-1939)

Resumo: A existência mesma dos impressos depende da vida e da obra dos livreiros, impressores e editores. Nas primeiras três décadas do século XX, em Montevidéu, graças á combinação de vários fatores, alguns destes agentes fizeram sua contribuição para afirmar uma cultura letrada débil e bamboleante. Dentre eles, figura fundamental foi o italiano Orsini Bertani (1869-1939), quem de notório anarquista no seu país de origem, na França, Inglaterra e Argentina tornou-se sossegado, fraterno e decisivo empresário cultural na capital uruguaia. Perguntar-se sobre seus dias e tarefas ajuda á sua comprensão e a comprender a cultura da sua época.

Palavras-chave: Vida de Orsini Bertani; Impressos e atividade cultural; Uruguay (1900-1939)

\begin{abstract}
The existence of the printed matter depends on the life and work of booksellers, printers and editors. In the first decades of the 20th century, in Montevideo, thanks to a combination of several factors, some of those agents contributed to assert a literacy culture weak and wobbly. Among them, crucial figure was the italian Orsini Bertani (1869-1939) that active anarchist in their country of origin, in France, England and Argentina became a calm, fraternal and decisive cultural entrepreneur in the Uruguayan capital. Wonder about his days and chores helps to understand him and understand the culture of his time.
\end{abstract}

Keywords: Orsini Bertani's life; Prints and cultural activities; Uruguay (1900-1939) 


\section{Introducción}

Nada tendría de extraño que en el cambio de los siglos precedentes alguien emigrara al Río de la Plata desde la empobrecida y convulsionada Europa. Que ese alguien fuera un adolescente y proviniera de una familia burguesa del norte de Italia con un historial de activismo anarquista y que, a la postre, se transformara en el principal editor en el Uruguay del Novecientos, merece ser atendido más allá de la estadística o de la anécdota. En ese estadio subalterno, el de la colección de pequeñas historias más bien simpáticas, en general respetuosas pero en tono menor, se ha mantenido a la vida y la obra de Orsini Bertani, así como a su contribución en este y otros planos conexos de la vida cultural montevideana y aun rioplatense. Se diría que en ese mismo estadio se ha considerado -si acaso se llevó adelante tal operación- el paso por el mundo de cualquier editor, librero e impresor hasta que, en el curso de los últimos años, la cultura material y sus efectos sobre las formas empezaron a observarse desde otras perspectivas.

En este trabajo, inserto en ambicioso y largo proyecto, ${ }^{1}$ sólo se trazará una semblanza, es decir apenas un retrato de un sujeto que no servirá sólo de pretexto para tratar de entender una época, tendencia cada vez más dominante entre los investigadores del campo histórico y cultural. En tan pocas líneas esta sería una aspiración presuntuosa, mucho más a sabiendas de que todo relato sobre una vida (todo relato sobre las cosas de este mundo) es provisional. Y que un transcurrir tan rico, tan lejano y tan lleno de acontecimientos sólo puede ser registrado $-\mathrm{o}$, mejor, recreado- como hipótesis a partir de algunos hechos, hasta cierto punto verificables o probados. Entre las muchas formas en que para la dimensión pública Bertani podría ser valorado a la distancia está su militancia anarquista juvenil -que cada vez interesa más-y, por otro lado, su labor como agente cultural (impresor, librero, editor). Esta breve historia de su vida, que se nutre de documentos y testimonios, trata de analizar episodios posibles de quien ahora es sólo una imagen perdida en

\footnotetext{
1 La bibliografía contemporánea sobre "mediadores culturales", como los llamó Robert Darnton (2010, p. 150), crece en todas partes en los últimos años. La perspectiva que buscamos ensayar ante este y otros casos de impresores y editores, sobre todo, tiene que ver con la relación entre actos de vida individual y colectiva, producción material e incidencia sobre las formas de escritura. (Cf. ROCCA, 2012). A comienzos de 2004 este trabajo se llevó a cabo a pedido del Sr. Francesco Piccinini, secretario de Cultura de la Comune di Cavriago, por gentil mediación de la Mag. Clara Aldrighi. Una primera versión fue entregada a fines de ese año según lo convenido y hasta el presente no se ha publicado en italiano, lengua a la que, según se me informó, fue oportunamente traducido. El 26/IV/2012 presenté parte de este texto en una conferencia en el Istituto Italiano di Cultura, de Montevideo. La investigación sobre Bertani, entre otros libreros, impresores y editores que se remontan a comienzos del siglo XIX, se inserta en el proyecto de Dedicación total "Las formas y sus medios", que llevo adelante en la Facultad de Humanidades y Ciencias de la Educación (Universidad de la República, Uruguay).
}

una época (1900-1939), un conjunto de signos, recuerdos e interpretaciones; por eso este relato procura atender con los mayores equilibrios esas dos grandes zonas incorporando otros casos ignorados sobre una existencia trashumante. Las aventuras de Orsini Bertani con sus inevitables matices e imponderables claroscuros trasmiten, en su apreciación global, una energía luminosa que deja la sensación de que sin su aporte y su personalidad otro hubiera sido el hilo de la cultura letrada moderna en el Río de la Plata. Bajo esa "empatía mínima" -el sintagma le pertenece a François Dosse (2007, p. 71)- se tratará aquí de acercarse a este sujeto extraordinario, ahora un "objeto" de estudio, simple colección de recuerdos de otros que se apagan, puñado de fotos buscadas afanosamente, signos impresos propios y, en especial, impresos ajenos que se juntan en su obra como editor, su imborrable legado.

\section{Europa, Europa}

Según consta en el Atti de nascita № 2.180, Orsini Menotti Bertani Toni nace en Firenze, el 26 de julio 1869 , "a ore cinque e minuti trinta". Son sus padres Eugenio Bertani (nacido en Cavriago el 17 de octubre de 1842) y Clementina Toni. Por datos que nos proporcionara el $\mathrm{Sr}$. Francesco Piccinini, el matrimonio tiene los siguientes hijos: Alberto Cavour (Cavriago, 17/II/1868); Mazzini (Roma, 17/III/1873), Garibaldi (Cavriago, 2/IX/1883) y Saffi (Cavriago, 2/XII/1880). "Come vede -comenta Piccinini- Eugenio diede ai suoi figli nomi di personaggi del Risorgimento italiano. Anche lui, prima di partire per l'Argentina fu fra i più importanti esponenti socialistianarchici di Cavriago e trasmise ai figli, assieme ai nomi, la passione per la politica e per l'impegno culturale e sociale. Alberto e Orsini, pur molto giovani, si erano già distinti come i più attivi del movimento socialista locale". ${ }^{2}$ Orsini era el apellido del revolucionario italiano que en 1858 atentó contra Napoleón III. Un destino que parecía marcado.

Cumplidos sus estudios primarios y secundarios en Italia, hacia 1885 Orsini fue enviado a París por su padre para que cursara la Universidad, según testimonio que en 2004 recogimos de su sobrina nieta, la Sra. Elisa Larré Bertani. ${ }^{3}$ Pronto, sin embargo, en territorio francés Bertani adhiere a las ideas anarquistas y sustituye la vida académica por la política radical. Según Luce Fabbri, quien trató de cerca a Bertani en la última década de su vida, por ese entonces participó de la banda anarquista que encabezaba Jules Bonnot, por cuyas actividades se

\footnotetext{
2 Comunicación electrónica remitida al autor de este artículo por el Sr. Piccinini, desde Cavriago, el 5 de febrero de 2004.

3 Entrevista a las señoras Perla Bertani y Elisa Lagouardette, 22 de abril de 2004, en el apartamento de la Sra. Bertani en la calle José Ellauri 868, Montevideo.
} 
pidió su captura. Antes de que la policía pudiera prenderlo Bertani huyó a Londres, donde vivió por muy breve lapso, donde - de acuerdo a una versión dudosa, que veremosconoció a Kropotkin. Otro testimonio agrega que Bertani salió de Inglaterra hacia Italia, donde también pasó una efímera temporada (FABBRI, 1996, p. 179).

Manuel de Castro informó algunos datos no del todo confiables sobre el editor de su primer libro, la novela Historia de un pequeño funcionario (1928), uno de los últimos títulos a los que Bertani acogió con su sello y su financiamiento. Puede suponerse que el agradecido Manuel de Castro de la madurez mitificó la memoria de su juventud bohemia en la que admiró a aquel hombre bueno y algo aventurero. Parece claro que toda información debió obtenerla del propio Bertani o de algún viejo anarquista italiano o argentino del Montevideo de los veintes o de los muchos relatos que hacían del librero y editor una leyenda viva. A pesar de sus errores ahora comprobables, este testimonio interesa si se maneja la hipótesis de la autobiografía oral construida en ámbitos fraternos por Bertani para amigos uruguayos jóvenes, privados de cualquier posibilidad de verificar ese pasado, para ellos física y temporalmente muy remoto:

Orsini Bertani era hijo de una acaudalada familia italiana, habiendo sido educado con sumo esmero en París y Londres. En esta última ciudad, trabó relación nada menos que con Kropotkhine, padre espiritual del anarquismo, figura venerable, con su barba de santo natural y cuyo pensamiento tanto influyó en las generaciones ácratas de principio del siglo [...] De regreso a París, bien pertrechado de conocimientos e idealismo anárquico, dióse por entero a la acción revolucionaria con tanta fogosidad y denuedo que durante un movimiento de reacción gubernamental fue instado a abandonar el territorio francés. Al salir su efigie en los diarios, se organizó una demanda en favor del expulsado, ganando la simpatía de los parisienses, en virtud de ser Bertani, "un morocho, muy buen mozo". Este episodio da un cariz novelesco a su turbulenta juventud.

Vuelto a su país de origen, habiendo llegado la edad de hacer el servicio militar, y amparándose en el concepto de ácrata de Hamon de que "el militarismo es la escuela del crimen”, se embarcó para Buenos Aires con su padre, donde a poco de instalarse, recomenzó su propaganda idealista, fundando el periódico revolucionario El Perseguido, donde colaboraba asiduamente Florencio Sánchez [...]" (CASTRO, 12/ $\mathrm{VI} / 1955)$.

Fuera de los pasos perdidos del sujeto mítico, no hay discusión acerca de que Bertani adhirió con firmeza a los ideales libertarios. Por eso, pronto y aún adolescente, no tuvo otra alternativa que salir de Europa, territorio demasiado peligroso para estas adhesiones y mucho más para sus prácticas.

\section{Buenos Aires}

A fines del siglo XIX la capital argentina era una ciudad dinámica, con un crecimiento que parecía imparable, donde había un altísimo porcentaje de inmigrantes italianos. Para 1895 estos llegaban a ser la mitad del $25,5 \%$ de extranjeros en el total de la población del país, uno de los guarismos más significativos en el mundo (DEVOTO, 2004, p. 49). Buenos Aires también tenía un número relevante de grupos anarquistas. Quizá por esto, hacia 1886, con apenas 17 años de edad, Bertani la eligió como destino. El propio Errico Malatesta, enfervorizado con el proyecto de organización social que había diseñado en Firenze en Questione Sociale, pudo idealizar Buenos Aires involuntariamente fiel a una tradición que remitía al pasaje de Giuseppe Garibaldi por el sur de Brasil y por Montevideo, cuyas actividades liberales continuaron resonando a un lado y otro del océano con enormes proporciones simbólicas para la comunidad italiana. En la capital argentina Bertani continuará en actividad dentro del socialismo anárquico vinculándose pronto a las figuras más notorias, en especial con sus compatriotas. En carta de Luigi Fabbri a Malatesta, datada en Montevideo el 17 de enero de 1930, aquel evoca el temprano conocimiento del líder anarquista italiano con Bertani, "propio quello del Perseguido di verso il 1894, mi pare. Ma lui mi dice che t'ha conosciuto anche prima, quando tu eri a Buenos Ayres, verso il 1886. Si dice sempre con noi nelle idee, e a me ha detto che non ha più que preconcetti individualisti e antiorganizzatori di una volta". ${ }^{4}$

Según parece, Orsini está poco tiempo solo en Buenos Aires porque, luego de la repentina muerte de su hermano Alberto Cavour (1887), toda su familia se reunirá con él en América. ${ }^{5}$ De acuerdo al ya citado Piccinini, el funeral de Alberto Cavour fue el primero en Cavriago que no estuvo presidido por un sacerdote y su prematura muerte fue "occasione di una grande manifestazione politica con oltre 2.000 persone; in sua memoria il Circolo Socialista eresse anche un monumento". Don Eugenio Bertani lleva consigo a Buenos Aires un importante capital que le permite instalar un negocio de importación de alimentos de origen peninsular, que prosperará. El membrete de una carta remitida a su nuera desde Buenos Aires a Montevideo, el 4 de noviembre de 1909, revela que el comercio se llamaba "Eugenio Bertani e hijo", y se especializaba en "Venta de comestibles por mayor y menor. Importación

\footnotetext{
4 Este documento como todos los que provienen de archivos italianos fueron obtenidos por la Profesora Mag. Clara Aldrighi, quien generosamente nos cedió copias de los mismos, así como su autorización para publicarlos.

5 Información proporcionada por la Sra. Perla Bertani.
} 
directa de aceites, quesos y conservas". ${ }^{6}$ La casa central se ubicaba en la calle Laprida al 518, y la firma contaba con cuatro sucursales en diversos puntos de la ciudad, dos de ellas en el febril Mercado de Abasto. No sabemos cuál de sus hijos se había incorporado como socio en la firma. Orsini, por entonces, ya llevaba varios años en la orilla oriental del Plata. Pero antes de cruzar el ancho río cabe conjeturar que resaltaba entre los integrantes de su familia por la vehemencia en la expresión de sus ideas. Esta opción pudo redundar en enfrentamientos con su padre quien, aunque liberal y garibaldino, estaba muy lejos de concebir a la propiedad privada como un crimen. Y sin embargo, todo da la pauta de que don Eugenio asistió económicamente a su hijo durante muchos años.

Orsini comenzó sus actividades ácratas en el periodismo, alrededor de los gremios de tipógrafos, los primeros sensibles a esta ideología. Ya era una figura destacada en estos medios cuando el 18 de mayo de 1890 comenzó a colaborar en El Perseguido, el periódico anarquista a que hace referencia Fabbri en la carta antecitada que, asediado por la censura y la represión, acabó siendo clausurado por las autoridades. Eran muchas las publicaciones anarquistas animadas por italianos. Con el antecedente de La Questione Sociale, de Malatesta, luego emergieron La Protesta Humana, y entre 1893 y 1894 "aparecieron publicaciones como La Riscosa, Lavoriamo y Venti Settembre, que luego se trasladó a Montevideo" (MANCUSO/MINGUZZI, 1999, p. 66). ${ }^{7}$ De gran número y de alta contribución para el pensamiento socialista rioplatense, estas publicaciones periódicas que florecieron a fines del siglo XIX, como ha señalado Luce Fabbri, eran obra de "autodidactos y no tienen avisos; sus temas principales son la lucha gremial y la organización sindical. Su lengua es algo vacilante y resiente el origen casi siempre extranjero de sus redactores" (FABBRI, 1993, p. 49).

En 1894 Bertani conoce a Elisa Lagouardette, nacida en Francia en un paraje rural entre Pau y Tarbes, quien había llegado a Buenos Aires -suponemos que de niñajunto a su familia católica y de buena posición económica. En esta ciudad se consuma el matrimonio. Allí nacerán tres de sus hijos: Adolfo Germán (muerto a los quince años), Alba y Verité.

\footnotetext{
6 Este y otros originales se encontraban en poder de la Sra. Perla Bertani en 2004.

7 Luce Fabbri registra tres publicaciones anarquistas con el nombre Venti Settembre en Buenos Aires. La primera editó un número único el 20 de setiembre de 1889; la segunda, hizo lo propio en idéntica fecha pero de 1895; la última, salió en la misma fecha de 1901 como suplemento de L'Avvenire (FABBRI, 1993, p. 60). Ni Fabbri ni otros investigadores de la prensa periódica en Montevideo (SCARONE; ZUBILLAGA/BALBIS, 1986, p. 97-112; Rodríguez/Ruiz) -ni quien esto escribe- encontraron publicación de este nombre en los acervos públicos o privados uruguayos con el mismo nombre.
}

\section{Montevideo}

Algunos de los documentos consultados dejan entrever que en fecha que no podemos precisar, pero que ronda el final de 1898 o comienzos del siguiente año, Orsini Bertani está en Montevideo. Su familia quedó en Buenos Aires. Es probable que entonces sólo haya pasado una temporada en la capital uruguaya para hacer algunas gestiones o explorar el ambiente y hacer los contactos necesarios con sus compatriotas o compañeros de causa; puede ser que regresara clandestinamente a Buenos Aires ya que sólo más tarde, en 1902, llegó a radicarse en la orilla oriental del Plata. Dos versiones, no del todo contradictorias, han circulado en múltiples testimonios escritos u orales sobre las razones de ese traslado último y definitivo. La primera, auspiciada por sus descendientes, indica que debió pasar a Montevideo como medida preventiva ante su inseguridad personal; la segunda, difundida en la prensa a pocos días de su muerte $-\mathrm{y}$, que sepamos, nunca rectificada públicamente por sus familiares-, señala que fue expulsado del territorio argentino por su militancia ácrata en 1902 al amparo de la Ley de Residencia (España Democrática, 31/III/1939; ZARAGOZA, 1996). Sea como fuere, a comienzos de esta fecha la familia entera ya estará reunida en la capital uruguaya.

Montevideo le ofrece otro tipo de seguridades. En principio se abocará a consolidar su numerosa familia. Allí, en pocos años, la pareja tendrá dos hijos más: en 1906, Perla de Oriente, y en 1907 Ibsen Delfos. En Montevideo desplegará su vocación y su amor por el universo de los impresos. Hacia 1903 en el corazón de la Ciudad Vieja, en la calle Sarandí N 240 casi Bartolomé Mitre, Bertani abre la Librería Moderna, que atiende junto a su esposa y a un empleado, Juan de Gregorio, futuro periodista y escritor, quien solía emplear el seudónimo David. A su vez, en momentos en que se incrementa la producción gráfica, el recién llegado monta la imprenta "El Arte" ubicada en la calle Reconquista $\mathrm{N}^{\circ} 195$, donde -según Manuel de Castro- introdujo la primera monotipo que se conoció en Uruguay, y en la que se hacían "Ilustraciones, Libros, Encuadernaciones, Trabajos comerciales, etc., etc.", como figura en el membrete de documentos emitidos por la firma. El momento no era el mejor. Durante casi todo el año 1904 una guerra civil asoló el país, que no afectó a Montevideo en sus operaciones militares pero sí en dificultades de toda clase, incluidas las económicas que como siempre primero llevan a la retracción del mercado de bienes simbólicos. Cuando en setiembre termina la guerra con la muerte del caudillo rival del gobierno, el general gaucho Aparicio Saravia, se abre una etapa de paz y de valorización progresiva de la cultura. Era la hora de Bertani. O eso pudo haber creído. 
De a poco, con ese carácter afable que le reconocen todos sus contemporáneos, Bertani se transformó en una especie de supremo sacerdote de varios escritores uruguayos emergentes. Solía encontrárselo en los cafés y las peñas de la bohemia literaria montevideana, especialmente el Polo-Bamba (LASPLACES, 1941; FERREIRO, 1945; ROCCA, 2006). No debe haber faltado en las mesas del cercano Café Tupí-Nambá, que en la época estaba frente a la Plaza Independencia, a pocas cuadras de su primera librería (MORA GUARNIDO, 1959). Tentado por la bohemia literaria pero, a su vez, urgido por una familia numerosa, Bertani probó suerte en algunos rubros asociados a esta afición. Por eso, hacia 1905 fundó una tipografía llamada La Merghenthaler, que funcionó en la calle Buenos Aires № 358.

La Librería Moderna fue su primera apuesta en el ramo que, durante unos años, convivió con otra casa de su propiedad, ubicada en la "Ciudad Nueva", en 18 de Julio № 342, esquina Convención. Se trata de la Librería Florencio Sánchez, inaugurada en 1911 a pocos meses de la inesperada muerte del joven y ya célebre dramaturgo, fallecido en Milán el 7 de noviembre de 1910. Tan profunda fue la amistad y la admiración de Bertani por Sánchez, a quien debió conocer en Buenos Aires, que alojó en su hogar por un tiempo a la viuda Catalina (Catita) Raventós, hecho que no suelen registrar los biógrafos del escritor. Hacia 1915 Bertani vendió la Librería Moderna al poeta Manuel Pérez y Curis (CASTRO, 1955; SILVA VALDÉS, 1959), para concentrarse en la segunda de sus casas, por lo menos durante dos años más. En ese momento ocupaba la mayor parte de su tiempo en la imprenta dejando la atención al público de la Librería en manos de la Sra. Elisa Lagouardette y de su fiel empleado Juan de Gregorio. Delmira Agustini, quien vivía a seis cuadras de este comercio, lo visitaba con frecuenta en procura de libros y -según testimonio de la Sra. Perla Bertani- para practicar su francés y para hacer confidencias íntimas a Elisa, las que con ella se han ido para siempre. Bertani editó toda la obra poética en vida de Delmira Agustini: El libro blanco, en 1907, Cantos de la mañana, en 1910 y Los cálices vacios, publicado en 1913, un año antes de que su exmarido la asesinara en una oscura pieza de la calle Andes, a otras seis cuadras de la Librería de sus amigos, esta vez en dirección sur, hacia el Río de la Plata.

Por confesión del propio dependiente sabemos que en la Librería Florencio Sánchez el singular propietario "implantó el sistema de venta de libros en remate, que se realizaban al atardecer y por la noche", los que podían prolongarse "hasta pasada la hora veinticuatro, llenándose la librería abarrotada de personas interesadas en adquirir volúmenes a precios rebajados, que caían bajo los martillos de los rema- tadores Barros y Montauti”. De Gregorio recuerda, además, que

Entre los asiduos concurrentes al remate y que permanecían además en la librería para conversar con Bertani, una vez que la gente se había retirado, hallábanse, Leoncio Lasso de la Vega, Alberto Lasplaces, José P. Bellan, [Vicente] Basso Maglio, [Carlos] Sabat Ercasty, Cyro Scoseria, Julio y Enrique Casaravilla Lemos [...] Iba también a la librería Alberto Zum Felde [...] (DE GREGORIO, 1945).

Antes, en 1904, con la publicación del volumen de poemas Cantos augurales, de Álvaro Armando Vasseur, el poeta uruguayo-francés de simpatías anarquistas, comenzó sus actividades el sello editorial O. M. Bertani. La oficina de la editorial estaba situada en la calle Reconquista al 630, en plena Ciudad Vieja de Montevideo. En apenas tres años, mientras declinaba la labor de otros colegas vinculados a las clases altas o al gusto entre romántico y realista (Barreiro y Ramos, Dornaleche y Reyes, Vázquez-Cores), Bertani se convirtió en el principal editor montevideano del Novecientos. Sin desprenderse de sus demás actividades (librero, impresor), apostó por la formación de un catálogo nuevo y ajeno al financiamiento de los autores, por lo que se consagró como el primer editor puro, con pretensiones de profesionalidad y promoción de líneas generales de escritura. No todo, es cierto, lo hizo por gentileza o por incontaminada devoción por la literatura nueva. Julio Speroni Vener afirma que Bertani coeditó con Claudio García algunos títulos "amarillos", a menudo de autores extranjeros, para consumo masivo: novelas policiales o sentimentales, aun aquellas que sobrepasaban los límites del pudor burgués (SPERONI VENER, 1989). Pero entre los títulos que llevan el sello O. M. Bertani no hemos podido encontrar ninguno que comparta el crédito con Claudio García, quien por 1910 puso la librería "La Bolsa de los Libros", un lustro después convertida en pujante editorial, aunque con otras características (ROCCA, 2005).

Varios de sus autores lo frecuentaban en su librería -como se vio- o aun en su domicilio. Hacia 1915, según su hija Perla, quien contaba once años en esa fecha, los amigos más asiduos de la casa eran el narrador y dramaturgo José Pedro Bellan y una pareja de jóvenes novios, el patriarca de la crítica Alberto Zum Felde y la jovencísima Clara Silva, más tarde poeta y narradora, quienes se casarán en el propio hogar de Bertani. Esta red de encuentros superó los vínculos amistosos emanados de los lazos profesionales. Hubo otras formas públicas con las que Bertani exteriorizó su aprecio o su apoyo a escritores de su tiempo: firmó el libro homenaje a Florencio Sánchez el 31 de mayo de 1905; fue el principal animador de numerosos banquetes en honor de Álvaro 
Armando Vasseur (febrero de 1907), el mencionado Sánchez (1907 y 1909) y Manuel Medina Bentancort (octubre de 1907), para todo lo cual abrió un registro de adhesiones en su Librería Moderna; integró el comité de homenaje a Julio Herrera y Reissig una vez que se produjo su repentina muerte, en 1910; puso a disposición del comité que organizó el banquete en homenaje a Ernesto Herrera su "magnifico salón Excelsior" (setiembre de 1910). Simultáneamente a este multifacetismo de acento estético el editor no abandonó la militancia política o las formas cercanas del apoyo a los grupos que sintió afines: en 1905 ayudó a la revista anarco-comunista Futuro, dirigida por Edmundo Bianchi y Leopoldo Durán, con la promoción para suscriptores durante un año quienes recibirían de obsequio el ejemplar de Cantos augurales (1904), de Vasseur, al mismo tiempo que financió avisos de su librería y editorial en esta publicación periódica.

Entre los más cercanos escritores al editor estaba Javier de Viana, aunque era de filiación blanca, devoto admirador de Saravia, y declarado antibatllista. Una abundante correspondencia, de la que sólo se ha dado a conocer las piezas de Viana, muestra el grado de confianza entre autor y editor se incrementa con el tiempo. Las estrecheces económicas del escritor radicado en Buenos Aires hacia 1910 encuentran en Bertani algo más que un interlocutor; encuentran una mano fraterna para solucionar problemas perentorios: "En cuanto a nuestros intereses -le escribió el 25 de setiembre de 1911-si Ud. precisa algún dinero, hágamelo saber que haré lo posible para enviarle algo [,] puesto que ya estamos bastante adelantados" (Museo Histórico Nacional, Tomo 153, folio 41). "Yo estoy asquerosamente pobre-le diría Viana desde Buenos Aires, el 13 de julio de 1914-. ¿Podría Ud. -sin sacrificio-, proporcionarme a mi llegada ahí algún dinero?". Y un mes más tarde, el 21 de julio, el escritor agradece: "El consentimiento del auxilio expresado en su telegrama, me ofreció la oportunidad de orillear yendo allí-, la peligrosa situación económica -vísperas de naufragio-, en que me encuentro" (Correspondencia de Javier de Viana..., 1972, p. 29-30). Sin rozar cimas tan dramáticas, el poeta Julio Herrera y Reissig también recurrió a Bertani en circunstancias difíciles, como lo muestra la carta del 14 de noviembre de 1909 en la que le pidió "algunos recursos, a cuenta de los resultados de mi próxima obra [...] Como juzgo que se ha olvidado y creo a usted un verdadero caballero y amigo -sin invocar para más nada las razones comerciales- espero se digne resolver sobre el particular, para lo que autorizo a nuestro común amigo Víctor Cardoso". 8

\footnotetext{
8 Original en un folio manuscrito. Colección Julio Herrera y Reissig, Archivo Literario del Departamento de Investigaciones de la Biblioteca Nacional. En cambio, las seis cartas o misivas enviadas por Roberto de las Carreras a Bertani, todas ellas sin data, tienen que ver con el pedido
}

La entonces indisociable actividad de librero y editor, auspiciada por su fino trato con los intelectuales y los clientes de la ciudad, pronto lo tornaron una figuranexo para las relaciones culturales. Así, por ejemplo, durante la visita a Montevideo del poeta Rubén Darío, ocurrida en 1912, este se encontró con la poeta Delmira Agustini gracias a sus buenos oficios: "Tal entrevista fue promovida por el propio editor Orsini Bertani, en casa de Delmira Agustini [y] sólo asistieron a la misma, la señora de Bertani, los familiares de la poetisa y Julieta de la Fuente de Herrera y Reissig" (CASTRO, 22/IX/1960).

El 4 de agosto de 1910 el editor le escribe a Viana planteándole, por primera vez, la intención de "hacer una revista" (Archivo del Museo Histórico, Tomo 152, folio 109). Un bienio después, ante la pregunta sobre esta iniciativa, comenta: “¿La revista? Es un tema que quema. La mayoría de los suscriptores quieren pagar portecipado [sic: ¿por anticipado?]- no hay modo de convencerlos de que se trata de una revista con vida asegurada, con una dirección seria[,] etc. etc.” (Museo Histórico Nacional, Tomo 153, folio 65). Se trata, seguramente, de La Semana, que sale en Montevideo entre 1909 y 1913, dirigida por Orestes Acquarone, aunque la dirección intelectual recayó en Alberto Lasplaces. En esta aventura a Bertani le tocó la peor parte, la del distribuidor, aunque nunca figure en los créditos, como lo aclara en otra carta dirigida a Viana el 24 de febrero de 1912.

A una década de su radicación en Montevideo $\mathrm{y}$ de haber intentado muchos, sucesivos y a menudo simultáneos negocios los réditos económicos no eran muy halagüeños. En 1910 instaló un cine, el Excelsior, situado en la calle 25 de mayo № 262; dos años después aún ofrecía funciones "todas las noches. Los domingos $y$ días festivos grandes matinés" (La Semana, № 164, 19/X/1912). Aparentemente el cine no se mantuvo mucho tiempo en sus manos, ya por una fallida administración, ya por quebrantos económicos de sus otros emprendimientos: "Para atraer a la gente, mi padre repartía bombones en la puerta. Yo era muy pequeña, pero tengo esa imagen y, además, en mi casa esa historia se repetía mucho" (Testimonio de Perla Bertani). ${ }^{9}$

De acuerdo con innumerables testimonios, la política dadivosa de Bertani fue la causa fundamental para la fragilidad de sus empresas. En rápido balance de los editores montevideanos del Novecientos, Zum Felde afirmó que Bertani “en la mayoría de los casos editaba

\footnotetext{
de que se encargara de importar papel de Holanda para la edición de un libro, seguramente Suspiro a una palmera, y el reclamo del envío de algunos títulos franceses e italianos de la librería de Bertani. (Colección Roberto de las Carreras, Archivo Literario, B. N.).

9 No era nada anormal la práctica de repartir bombones, y hasta juguetes, como es obvio entre el público infantil. Así se promocionan las matinés del Biógrafo Yris, de la calle Arenal Grande en la misma sección de avisos de La Semana.
} 
desinteresadamente, por amor a la intelectualidad y a la cultura; ni editados ni ediciones reintegraban, en general, el costo de imprenta. Y si a esta generosidad de editor se agrega su generosidad de amigo, se comprende que, en vez de prosperar, al cabo de un tiempo, imprenta y librería se arruinaran" (ZUM FELDE, 1930). Entre tantas voces de elogio hay una que disuena. Se trata de la viuda de Rafael Barrett, muerto prematuramente en Asunción del Paraguay luego de un pasaje consagratorio por Montevideo. En el prólogo a la correspondencia con su marido se queja de su editor: "El único libro que dejó hecho [Barrett] fue Moralidades actuales. Después mandé editar yo los otros doce. Bertani nunca rindió cuentas, haciendo varias ediciones" (Cf. BARRETT, 1961, p. 9). ${ }^{10}$ Como sea, minaba sus energías la lucha cotidiana con los clientes reales más que con los bohemios que lo asediaban para que les publicara sus versos o sus prosas. Ese desgaste constante se lo hace saber a Javier de Viana, siempre asfixiado por sus propias cuentas:

Para darle una idea de cómo pasan las cosas, le citaré algunos casos ocurridos con personas que Ud. conoce:

-Díaz, el sombrerero, contesta a mi cobrador que no le ponga más los pies en la casa que él pagará cuando le de la gana y nunca anticipadamente.

-Stábile, el peluquero, contesta que no paga más de 0.40 por mes y se extraña, etc. etc.

-Pelayo, el diputado, contesta que Viana es un amigo suyo y que eso de irle a cobrar es una indecencia.

En fin, en un mes que tengo en la calle, mi dependiente corre como un endemoniado todo el día, y hasta ahora no ha alcanzado a cobrar ¡80 pesos!" (Carta del 24 de febrero de 1912. Museo Histórico Nacional, Tomo 153 , folio $68-b)$

Algo acorralado por las deudas, para reducir costos en 1914 Bertani trasladó la editorial a la calle Arapey (hoy Río Branco) № 1423, que en el momento coincidía con el domicilio familiar, una amplia casa a la que debió robarle al menos una habitación al frente para sus menesteres comerciales. Así se fue esfumando la ilusión de una cultura para todos. Por si hiciera falta alguna prueba hay una carta estremecedora del 8 de diciembre de 1911, dirigida a su amigo Viana en la que comunica

\footnotetext{
${ }^{10}$ Sin embargo, en cartas a su mujer y luego prologuista, en sólo dos ocasiones Barrett menciona a Bertani, en ninguna de ellas de modo duro o recriminatorio: 1) "[...] por fin, el editor Bertani de Montevideo está en tratos para editarme un libro que tengo que preparar en 6 meses" (Carta fechada el 1o de marzo [de 1909]). 2) "Vi a Frugoni, a Falco, a Bertani -que me ha pedido originales para otro libro-, a Herrerita, a reporters de toda laya, directores de revistas, fotógrafos (;me retrataron 3 veces!), un escultor me quiere hacer el busto -los melenudos del Polo Bamba- y los que más me agradaron, obreros, tipógrafos, jornaleros que me llamaban 'maestro' y me estrujaban las manos entre las suyas callosas" (Carta del 11 [de setiembre de 1909], comentando su partida de Montevideo). (BARRETT, 1961, p. 73; 98).
}

su resolución de "retirarme de mis actuales negocios". Y agregaba: "Créame, mi buen amigo, no hay esclavitud peor que la que sufre el pequeño industrial. Se es humilde, se es mendigo, se es industrial al fin, y no hombre". ${ }^{11}$

A la larga, Bertani venció. Porque un número altísimo de autores y textos editados por Bertani pasaron a formar parte del canon literario uruguayo y latinoamericano: Herrera y Reissig, Sánchez, Viana, Ernesto Herrera, Delmira Agustini, Vasseur, por lo menos. En aquel entonces sólo el dinero del negocio de su padre, que se volcaba hacia Orsini en situaciones de emergencia, hacía posible la reconstrucción de los lances culturales montevideanos y el renacimiento de la fe. Ese dinero también propiciaba otros emprendimientos más curiosos.

\section{Trayectos públicos}

Como tantos otros uruguayos y extranjeros de la misma línea, hacia 1912 Bertani abandona la militancia anarquista y, sin desprenderse de sus supuestos básicos, adhiere al grupo político que orienta en el Partido Colorado el presidente de la República, José Batlle y Ordóñez. Dos documentos hablan de esa transformación de una ideología y la prolongación de una conducta política: en 1907 aparece integrando la comisión de respaldo al propagandista libertario Constante Carbalho, expulsado de Buenos Aires; en 1914 firma junto a notorios batllistas el "Comité popular pro reforma" constitucional. Más allá de sus complejidades podría convenirse que el primer batllismo ensayó una política reformista democráticoradical. Así caracteriza Luce Fabbri el vuelco político de Bertani: "era diventato amico ed ammiratore di Batlle e tutto il suo sistema mentale era cambiato, senza che egli cessasse di considerarsi anarchico" (FABBRI, 1996, p. 179). De hecho, hay algunas trazas del trato personal entre Bertani y el presidente Batlle y Ordóñez, ${ }^{12}$ quien en su segundo mandato lo designó Inspector de Trabajo, con certeza cuando zozobraban las actividades particulares de su seguidor. De ese modo, Batlle además se aseguraba la inclusión de los más díscolos en favor de su proyecto y, al tiempo que los cooptaba, podía alimentar su movimiento con ideas socialmente avanzadas que lo distinguían de otros grupos de su propia colectividad política y lo distanciaban de la mayoría del Partido Nacional, su adversario tradicional y más numeroso.

Una abundante documentación que nos permitiera consultar su hija Perla -fallecida mientras redacto la

\footnotetext{
11 Original en la Colección Javier de Viana, Museo Histórico Nacional, Casa de Lavalleja, Tomo 153. Citada en Rocca, 2012.

12 Perla Bertani conservaba en la mencionada fecha en que pudimos apreciarla una tarjeta con el membrete "José Batlle y Ordóñez. Presidente de la República". Debajo de esta inscripción, la frase manuscrita: " $\mathrm{Al} \mathrm{Sr}$ Orsini Bertani: Muy agradecido".
} 
última versión de este trabajo el 22 de mayo de 2017 , a la edad de 110 años- muestra que aproximadamente por 1909 Bertani se integró activamente a la Masonería, de la que, al menos en apariencia, comenzó a alejarse sólo hacia el final de su vida. Sobre sus primeros años en la logia nos ha quedado un testimonio privilegiado en la memoria de Alberto Zum Felde: "Bertani no escribía, pero era un espléndido orador. Yo le oí un magnífico discurso en una logia masónica" (VISCA, 1969, p. 17).

Aunque fuera originario de Italia, siempre estuvo involucrado en la vida política uruguaya, como antes lo había estado en Argentina y, si se quiere, en Francia. $\mathrm{Y}$ aunque concibió el mundo como tarea colectiva, un poco fuera de las parcelas nacionales, sintió la vida y los problemas italianos en especial desde que los acontecimientos que encumbraron al fascismo en 1922 colocaron a su país de origen en el centro de una tormenta ideológica internacional. Sin demoras, Bertani se convirtió en activo antifascista, más que nada por decidido enemigo del nacionalismo extremo y por su renovada fe en la libertad y su final confianza en las prácticas demo-liberales.

\section{Otros pasos}

A fines de 1915, cuando comienzan los aprietos en los negocios editoriales y el comercio de libros, la familia se muda a una casa de 21 de setiembre $\mathrm{N}^{\circ} 586$, casi Roque Graseras, en el barrio de Pocitos que, en la época, era un pueblo donde los sectores económicos acomodados solían veranear. Poco después Bertani hizo construir en el extenso fondo de la propiedad un gran galpón, donde alojó lo que quedaba de sus prensas y sus existencias bibliográficas, ya que en lo sucesivo apenas si publicó media docena más de libros hasta 1932. En esta última fecha sale el último volumen del que tenemos noticia al que presta su sello: los poemas en italiano de la joven hija de un querido amigo y paisano, I canti dell' atessa, de Luce Fabbri. La familia había ocupado diversas casas en el centro de la ciudad, y poco antes del traslado a Pocitos habitaron un amplio chalet en la zona de Tres Cruces, no muy lejos del centro y muy cerca del Hospital Italiano, por ese entonces una zona bastante despoblada.

Aun alejado del centro de las operaciones cotidianas de la vida cultural, Bertani no se apartó por completo de sus antiguas relaciones. Según Manuel de Castro siguió frecuentando los cafés literarios, en particular el Británico, en la ciudad vieja, lugar de encuentro de bohemios y anarquistas. Hacia 1920 dio un curioso paso. En la calle Cerro Largo, a pocos metros de Florida, instaló un restaurante popular denominado "La Cocina Económica". La finalidad de esta "empresa" era claramente social: facilitar una alimentación de buena calidad y de costo reducido a obreros, empleados e intelectuales con escasos recursos. Pero la experiencia duró poco, ya que el propietario solía distribuir fichas que permitían el consumo de los alimentos entre los artistas desocupados. El costo de estas fichas era ínfimo: ascendía a 2 o 5 centésimos (DURAND, 1980). Sólo para tomar un indicador corresponde reparar en que el precio de un volumen popular simple editado por Bertani ascendía, por ese entonces, a 40 centésimos. En el local, amplio e iluminado -según recordó su hija Perla- había una mesa con libros, con la finalidad de difundir la cultura letrada entre los trabajadores. Antonio Soto ("Boy"), recuerda que el título preferido -o casi el único- para ofrecer a los comensales era El Conde de Montecristo, de Alexandre Dumas, seleccionado como narración edificante $\mathrm{y}$ instigadora para los nuevos lectores, como le habría sido sugerido a Bertani por la lectura de un texto de Emilio Castelar. Manuel de Castro da su pintoresca versión de estas experiencias, a su parecer entrelazadas:

\begin{abstract}
Ocasionalmente, pero con cierta frecuencia, solía aparecer [por el Café Británico] Orsini Bertani, acompañado de Javier de Viana o del dramaturgo Ernesto Herrera, con su asma pertinaz y su característica camisa 'gorkiana'. Bertani había finiquitado su célebre editorial, donde encontraron acogimiento los destacados escritores uruguayos de principios del siglo, ya que el presidente Batlle con sutileza gubernamental al igual que lo hiciera con los anarquistas más connotados, lo nombró inspector del Trabajo. Pero nuestro hombre alternaba tal cometido (hoy se le hubiera juzgado por implicancia), con la Cocina Económica, que fundara en un viejo barracón de la calle Ciudadela, convenientemente adaptado a tal efecto y donde se daba de comer a 400 personas por día y ¡a cinco centésimos el plato! Orsini Bertani andaba siempre con los bolsillos repletos de fichas (válidas por una consumisión en comida), las cuales repartía gratuitamente entre los bohemios del Café Británico y al primer amigo que encontraba por la calle. De esta manera el negocio no duró mucho tiempo, aunque persistió un par de años (CASTRO, 13/III/1955).
\end{abstract}

\section{La última aventura}

Luego de varios años de mínima inactividad editorial, en 1927 Bertani decidió emprender su última gran aventura con la publicación de la revista cultural La Pluma. En ella se combinan textos literarios, de acuerdo al modelo convencional de revista de estas características, con una nítida orientación política, liberal radical en lo americano y claramente antifascista en términos generales. Por ofrecimiento de su propietario, la dirección intelectual recayó en Alberto Zum Felde, quien ya contaba con un largo desempeño en las páginas culturales de la edición 
vespertina de El Día. En 1971, en una entrevista ofrecida a Jorge Ruffinelli para el semanario Marcha, Zum Felde reconstruyó su contacto con Bertani reconociéndole, antes que nada, la exclusividad en la creación del proyecto: "De él fue la idea de hacer una revista, no mía. Él la financió (como algo muy novedoso en la época, y muy revolucionario, la financiaba con avisos que él mismo conseguía de sus amistades). Yo me limité a la dirección general de la revista [...]" (RUFFINELLI, 1985, p. 29).

En la biografía de Luigi Fabbri, quien arribó a Montevideo con su familia en 1929, Hugo Fedeli caracteriza a La Pluma como una "bellisima e lussuosa rivista artistico-letteraria di avanguardia che pubblicava già da qualche tempo a Montevideo un ex compagno nostro, Agostino (sic) Bertani, persona molto simpatica ed attiva" (FEDELI, 1948, p.92). En realidad, después de vivir el exilio en Francia y en Bélgica, Fabbri recibió un apoyo fundamental de Bertani quien, como recordó la hija del teórico anarquista, llegó a obsequiarle para su casa montevideana un "armadio, stretto ed altissimo, con un enome specchio, regalo di Orsini Bertani, un quasi compagno di cui non ho ancora parlato, ma che ebbe allora molta importanza per noi" (FABBRI, 1996, p. 178). Por su lado, Luigi Fabbri en 1929 había enviado a Malatesta sus primeras impresiones sobre los paisanos y compañeros con que se encontró en sus primeros días uruguayos, entre ellos destacaba al "vecchissimi amici di qui -non so se a suo tempo erano quí, o a Buenos Ayres- [...] el Sig. Orsini Bertani, ispettore del lavoro alle dipendenze del governo di qui, da queste parti giá da piú di 40 anni, ma che ha girato mezzo mondo e ti ha conosciuto anche lui 30 o 40 anni fa" (FABBRI, 1929). En la precitada carta a Malatesta, de 1930 completa un retrato de su viejo amigo:

Ma è ritirato da ogni cosa già da moltissimi anni, ha rotto ogni rapporto col movimento e anche personalmente con molti compagni. Alcuni di questi mi dicono che um tempo era molto cattivo (como individualista) versi di noi, che maltrattò molto [Pietro] Gori ecc. ma ora a me pare una vera buona pasta d'uomo, di ottimo cuore e sempre disposto ad aiutare um amico. Egli è ispettore del lavoro di questo governo; e quando un amico è disoccupato lui si fa in quattro per trovargli lavoro e ci riesce quasi sempre. Personalmente poi è anche editore di una grande rivista letteraria e artistica, Pluma (sic) fatta all'americana, piena di annunzi ma estranea o quasi alla politica. Io ci ho scritto un articolo per eccezione (FABBRI, 1930).

Por esos días, un conjunto significativo de intelectuales uruguayos (o radicados en Uruguay), organizaron una reunión en homenaje al "editor-artista", según lo llamó en la ocasión la escritora española Mercedes Pinto. Como forma de perpetuar el reconocimiento, encabezados por esta escritora radicada entonces en Montevideo, estamparon su firma y algunos agregaron unas palabras de gratitud, como Zulema Bautista Alcaraz de Dieste, Luis Eduardo Pombo, Bartolomé Firpo y Firpo, Adolfo Montiel Ballesteros, Humberto y Francisco Frangella, Manuel de Castro, Antonio Pena, entre muchos otros. El homenaje también puede apreciarse como despedida a una empresa y a un creador de cultura que estaba haciendo sus últimos esfuerzos en un medio que, más allá de los deseos y las euforias, apenas respondía a las expectativas creadas.

Paralelamente, sus pasos no eran ignorados por los diplomáticos fascistas en Montevideo. Tanto que, en 1932, el representante del gobierno de Mussolini cree relevante informar a su Ministro que "Il volumetto [di Luce Fabbri] en parola dal titulo I canti dell'atessa, sarebbe stato editato da un tal BERTANI, pure anarchico, tipografo, con stamperia in Montevideo, forse Calle Juncal № 1527, ed abitante in Calle 21 de Septiembre № 586 della predetta cittá” (Regia Ambasciata, 1932).

\section{Final y sin treguas}

Cuando el 31 de marzo de 1933 el presidente Dr. Gabriel Terra disuelve las Cámaras (JACOB, 1985), la dictadura saca a Bertani del puesto de Inspector en la Oficina Nacional del Trabajo "y lo manda a [la ciudad de] Trinidad como una forma de sanción", por sus visibles posiciones contrarias al nuevo régimen (Entrevista a Perla Bertani). El desplazado funcionario permanece en la pequeña ciudad, a centenar y medio de kilómetros de la capital, durante todo el año hasta que se acoge al beneficio jubilatorio. En diciembre de ese año terrible, el anarquista Gaetano del Massa, apresado por la policía cuando intenta ingresar a Italia, cautivo, enfermo y seguramente torturado, se transforma en informante del régimen de Mussolini. En el patético relato de su delación se destaca la actividad antifascista de Bertani:

\footnotetext{
Difatti, come ho detto, il 31 dicembre $1931 \mathrm{mi}$ imbarcai sul Piroscafo Inglese 'Highaland' (sic) diretto a Montevideo. [...] Oltre il Gabbr e il Fabbri ho consciuto, pure a Montevideo, tal Rasenti negoziante di scarpe; Bertani, toscano, ispettore dell'Officina del Lavoro $[\ldots]$

Verso la fine del 1932 fu tenuta a Montevideo un comizio dalle On/le Parini Segretario Generale dei Fasci all'Estero. In tale occasione pero opera del Bertani furono stampati e distribuiti numerosi manifesti antifascisti (R. Prefettura di Firenze, 1933).
}

En 1938, al borde de cumplir los setenta años, Bertani movilizó a un amplio conjunto de compatriotas italianos para fundar el "Círculo 'El Progreso'. Asociación Democrática, cultural y recreativa ítalo-uruguaya", 
cuya sede se ubicó en 18 de julio, № 1497 bis. Si bien en los "Propósitos" de esta institución se deslinda toda pertenencia formal a un grupo político determinado, pronto se llega una definición clara sobre los dos grandes campos ideológicos en pugna:

Los acontecimientos que desde tiempo se desarrollan en el mundo, demuestran que una sola línea de demarcación, bien pronunciada y definida, separa [a] los hombres en dos grandes y únicos grupos: uno, apoyado, sostenido y defendido por todas aquellas personas que pretenden restablecer regímenes sociales y políticos contra los cuales la historia, en sus luchas seculares, ha pronunciado sus sanciones definitivas; y el otro, a cuyo alrededor acuden todos los hombres progresistas - desde el más simple evolucionista hasta el idealista más avanzado- para allanar el camino que recorre la Democracia, a fin de que ella pueda completar su ciclo histórico de acuerdo con los modernos dictados de la Economía y de la Justicia Social (Estatutos, 1938, p. 4. Destacados en el original).

Un bienio después de su fundación, en 1940, el Círculo alcanzaba los dos mil socios (Progreso, marzo de 1940). En este espacio Bertani se desempeñó con la mayor energía, llegando a pronunciar a comienzos de 1939 una conferencia sobre Franklin Delano Roosevelt y el nazifascismo, en la que impugnó que este último representara "ideal alguno. La barbarie no puede ser alimentada por un ideal. Los fascistas quieren cerrar el paso a la democracia, porque la democracia es el gran camino que conducirá a los pueblos a su emancipación". De joven anarquista a convencido liberal: ese fue su claro trayecto.

Pocos días más tarde de pronunciada esa conferencia, el 17 de marzo de 1939, murió en Montevideo. Se lo veló en la sede del Círculo "El Progreso". Al día siguiente, sus restos fueron al Cementerio Central, donde realizó el discurso de despedida el poeta batllista Ovidio Fernández Ríos, uno de sus más cercanos amigos de los últimos tiempos. Según las informaciones de prensa y de acuerdo a las fotografías que acompañan varios de estos artículos, la concurrencia al sepelio fue multitudinaria. El 20 de marzo se le tributó un homenaje en la Cámara de Representantes por iniciativa del diputado batllista Julio V. Iturbide, con la adhesión del legislador socialista Emilio Frugoni, de quien Bertani publicó dos libros de poemas (El eterno cantar, 1907 y Los himnos, 1913). Los 33 diputados presentes se pusieron de pie (Diario de Sesiones, 1939).

El mismo año se editó una "Marcha fúnebre (para piano)", compuesta por Adriano Rodríguez y ofrendada "A la memoria del gran demócrata Orsini M. Bertani (Homenaje del autor)". La portada de este folleto de cuatro páginas incluye una fotografía del evocado, que fue tomada en la última época de su vida. Esa imagen muestra un rostro relleno en el que habitan un ancho bigote gris, una mirada vivaz, una sonrisa amplia. Levemente, la mano apenas entrecerrada del brazo derecho sostiene esa cabeza sobre la que se planta un borsalino. Completan el cuadro un traje claro, una camisa blanca, una corbata a rayas. Todo respira ganas de vivir.

\section{Referencias}

\section{Fuentes}

\section{Archivos públicos y privados}

Archivio Centrale dello Stato, Divisione Affari Generali e Riservati Casellario Politico Centrale (CPC), Roma: ${ }^{13}$

- Lettera de Luigi Fabbri a Enrico Malatesta, impostata a Montevideo il 18.II.29 indirizzata a Enrico Malatesta - Via Andrea Doria 97 - Roma. Datada en "Montevideo (Casilla de Correo 141), 15.II.1929. Archivio Centrale dello Stato (ACS), Casellario Politico Centrale (CPC), folio 1].

- Lettera de Luigi Fabbri a Enrico Malatesta. ACS, CPC. Datada en "Montevideo 17.I.1930. folios 2-3.

- Regia Ambasciata d'Italia al Regio Ministero dell'Interno Casellario Politico Centrale, Roma. ACS, CPC, b 1906, Ogetto: "FABBRI Luce di Lui i, anarchica". Datada en "Buenos Aires, li 19.9.1932"

- Firenze 19 Dicembre 1933/ R. Prefettura di Firenze. Div. U $\mathrm{P}^{\circ}$. - Prot. № 07299. Risposta a nota № 84121/90353. On/ le Ministero dell'Interno. Div. Affari Gen. e Riservati CPC, Roma. Oggeto: DEL MASSA Gaetano fu Luigi, folio 5.

- Archivo Literario. Departamento de Investigaciones de la Biblioteca Nacional, Montevideo, Uruguay. Colecciones Roberto de las Carreras, Ernesto Herrera, Julio Herrera y Reissig, José Enrique Rodó, Florencio Sánchez, Javier de Viana y Alberto Zum Felde.

- Correspondencia de Javier de Viana a Orsini Bertani publicada en Revista de la Biblioteca Nacional, Montevideo, n. 5, mayo de 1972, p. 25-32. (Presentación y notas de Arturo S. Visca).

Archivo del Museo Histórico Nacional, Casa de Rivera, Montevideo, Uruguay. Colección Javier de Viana, tomos 152 y 153. [Incluye 34 piezas remitidas por Orsini Bertani, algunas dictadas al escritor Manuel Pérez y Curis, entre el 4 de marzo de 1910 y el 15 de febrero de 1914. En su totalidad se encuentran inéditas].

Archivo de la Sección de Archivo y Documentación del Instituto de Letras (SADIL), Facultad de Humanidades y Ciencias de la Educación, Universidad de la República, Montevideo, Uruguay. Colección Manuel de Castro. Colección de recortes de prensa.

Archivo particular, Sra. Perla de Oriente Bertani, Montevideo, Uruguay. Consultado en 2004.

\section{Repertorios bibliográficos}

BARROS-LÉMEZ, Álvaro. La obra cuentística de Javier de Viana. Investigación bibliográfica y hemerográfica de la obra de Javier de Viana publicada durante su vida. Montevideo: Libros del Astillero, 1985. [Incluye parte de la correspondencia de Javier de Viana a Orsini Bertani antes publicada en la Revista de la Biblioteca Nacional, ver: p. 134-139].

\footnotetext{
${ }^{13}$ Documentación recabada por la Profesora Mag. Clara Aldrighi, cedida generosamente para este trabajo.
} 
FABBRI-CRESSATTI, Luce. Periodismo italiano en el Plata a partir de la Guerra Grande. En: Garibaldi, Montevideo, n. 8, p. 41-61, 1993. [Incluye dos Apéndices: "La prensa de la inmigración italiana en el Uruguay (hasta 1984)" y "Primer esbozo de una lista de publicaciones periódicas italianas en Argentina (hasta 1920)"].

RODRÍGUEZ, Mercedes; Ana María RUIZ. Bibliografía de la prensa periódica de Montevideo, 1906-1930. (Prólogo de Alfredo R. Castellanos). Montevideo, Instituto Nacional del Libro/Ediciones “El Galeón”, 1990.

SCARONE, Alberto. La prensa periódica del Uruguay [18961905]. En: Revista Nacional, Montevideo, n. 44, p.270-292, agosto 1941 ; n. 49, p. 71-99, enero 1942; n. 53, p. 386-403, mayo 1942; n. 60, p. 104-117, dic. 1942; n. 74, p. 279-294, feb. 1944.

\section{Documentos oficiales}

DIARIO DE SESIONES. XXXIII Legislatura - Segundo Período. Cámara de Representantes. 2ª sesión ordinaria, marzo 20 de 1939. Punto 7: “Orsini Bertani. Exposición de motivos y moción de homenaje del señor Representante don Julio V. Iturbide. Adhesión del señor Representante Emilio Frugoni, a nombre de la bancada socialista. (Aprobación de la moción Iturbide). Homenaje de la Cámara", en Diario Oficial, 14 de abril de 1939: 39-B - 41-B.

DIRECCIÓN GENERAL DE ESTADÍSTICA Y CENSO. Censo Comercial e Industrial de Montevideo. Montevideo, s/e, 1908.

ESTATUTO del 'CCírculo 'El Progreso'. Asociación democrática, cultural y recreativa ítalo-uruguaya”. Montevideo, s/e, 1938.

\section{Publicaciones periódicas relevadas}

El Día (diario), 1905-1929.

La Semana (revista), 1912-1913.

El País (diario), 1920-1930.

La Mañana (diario), 1925-1950. [Especialmente el Suplemento Femenino]

La Pluma, revista, 1927-1931, 19 v.

\section{Teoría, historia, crítica}

ALDRIGHI, Clara. Antifascismo italiano en Montevideo. El diálogo político entre Luigi Fabbri y Carlo Rosselli. Montevideo, Universidad de la República/Facultad de Humanidades y Ciencias de la Educación, 1996.

ALDRIGHI, Clara. Luigi Fabbri en Uruguay, 1929-1935. En: Estudios Migratorios Latinoamericanos, Buenos Aires, n. 37, p.389-421, 1997.

BARRÁN, José Pedro; NAHUM, Benjamín. Batlle, los estancieros y el Imperio Británico. Tomo I: El Uruguay del Novecientos. Montevideo: Ediciones de la Banda Oriental, 1978.

BERETTA, Alcides. Emigración italiana y modernización en la periferia uruguaya. Los italianos en la creación del sector industrial (1870/1930). En: Studi Emigrazione - Rivista trimestale del Centro Studi Emigrazione Roma, Roma, n. 150, p. 227-251, Giugnio 2003.

CLAPS, Manuel (colaboración de LAMAS, Mario Daniel). El batllismo como ideología. Montevideo: Cal y Canto, 1999.
DARNTON, Robert. [1990]. O beijo de Lamourette. Mídia, cultura e revolução. Trad. de Denisse Bottmann. São Paulo: Companhia das Letras, 2010.

DEVOTO, Daniel. Historia de la inmigración en la Argentina. 2. ed. Buenos Aires: Editorial Sudamericana, 2004. (Con un apéndice sobre la inmigración limítrofe por Roberto Benencia). DOSSE, François. El arte de la biografía. México: Universidad Iberoamericana, 2007. [Sin registro de traductor].

FABBRI-CRESSATTI, Luce. L'influenza della letteratura italiana sulla cultura rioplatense (1853-1915). Montevideo: Editorial Lena, 1967.

FABBRI-CRESSATTI, Luce. El aluvión inmigratorio italiano en el Uruguay de hace un siglo. En: Garibaldi, Montevideo, n. 5, p. 51-67, 1990.

FABBRI-CRESSATTI, Luce. Italianos en el Uruguay en las primeras décadas del siglo XX. En: Garibaldi, Montevideo, n. 6, p. 21-35, 1991.

FABBRI-CRESSATTI, Luce. Luigi Fabbri. Storia d'un uomo libero. Pisa: BS Edizioni, 1996. (Versión en castellano: Montevideo, Comunidad del Sur, 2001).

FEDELI, Ugo. Luigi Fabbri. (Prefazione di Luce Fabbri). Torino: Gruppo Editoriale Anarchico, 1948.

GARCÍA ESTEBAN, Fernando. [1937]. Vida de Florencio Sánchez. Montevideo: Alfa, 1970.

IMBERT, Julio. Florencio Sánchez. Vida y creación. Buenos Aires: Editorial Schapire S. R. L., 1954.

JACOB, Raúl. El Uruguay de Terra. Montevideo: Ediciones de la Banda Oriental, 1985.

MANCUSO, Hugo; MINGUZZI, Armando. Entre el fuego y la rosa. Pensamiento social italiano en Argentina: Utopías anarquistas y programas socialistas (1870-1920). Buenos Aires: Biblioteca Nacional \& Página/12, 1999.

MAROCCO, Gianni. Sull' altra sponda del Plata. Gli italiani in Uruguay. Milano: Franco Angeli, 1986.

ODDONE, Juan A. La emigración europea al Río de la Plata. Motivaciones y proceso de incorporación. Montevideo: Ediciones de la Banda Oriental, 1966.

ODDONE, Juan A. La formación del Uruguay moderno. La inmigración y el desarrollo económico-social. Buenos Aires: EUDEBA, 1966.

ROCCA, Pablo. Las ediciones populares de Claudio García (un proyecto cultural y su época, 1900-1945). En: Anuario del Centro de Estudios Gallegos, Montevideo: Universidad de la República/Facultad de Humanidades y Ciencias de la Educación/Centro de Estudios Gallegos, 2005. p. 87-108.

ROCCA, Pablo. Dos Cafés, una cultura urbana. Lugares del 'Polo Bamba' y el 'Tupí-Nambá': Los hermanos San Román. En: Anuario del Centro de Estudios Gallegos, Montevideo: Universidad de la República/Facultad de Humanidades y Ciencias de la Educación/Centro de Estudios Gallegos, 2006. p. 125-137.

ROCCA, Pablo. Editar en el Novecientos (Orsini Bertani y algunos problemas de las culturas material y simbólica). En: Orbis Tertius, v. 17, n. 18, 2012. ISSN 1851-7811. <http://www. orbistertius.unlp.edu.ar/article/view/OTv17n18d05/4892>.

SILVA, Clara. Pasión y gloria de Delmira Agustini. Buenos Aires: Losada, 1972. 
SPERONI VENER, Julio. Las ediciones furtivas de Claudio García. En: Revista de la Biblioteca Nacional, Montevideo, n. 26, p. 9-13, dic. 1989.

SURIANO, Juan. Anarquistas: cultura y política libertaria en Buenos Aires (1890-1910). Buenos Aires: Manantial, 2001.

ZARAGOZA, Gonzalo. Anarquismo argentino (1876-1902). Madrid: Ediciones de la Torre, 1996.

ZUBILLAGA, Carlos; BALBIS, Jorge. Historia del movimiento sindical uruguayo. Montevideo: Ediciones de la Banda Oriental, 1985-1992. (4 t.). [Especialmente: Tomo I, Cronología y Fuentes (Hasta 1905), 1985; Tomo II, Prensa obrera y obrerista (18781905), 1986; Tomo IV, Cuestión social y debate ideológico, 1992].

ZUBILLAGA, Carlos. Luchas populares y cultura alternativa en Uruguay. El Centro Internacional de Estudios Sociales. En: Siglo XX - Revista de Historia, Monterrey, Facultad de Filosofía y Letras, Universidad Autónoma de Nuevo León, n. 6, p. 11-39, jul.-dic. 1988.

ZUM FELDE, Alberto. Crítica literaria: Tres épocas. En: El Día - Edición de la Tarde, Montevideo, 17 jun. 1920. [Contiene una semblanza de Orsini Bertani].

ZUM FELDE, Alberto. Proceso intelectual del Uruguay. II. La Generación del Novecientos. Montevideo: Ediciones del Nuevo Mundo, 1967. p. 40-41.

\section{Testimonios, crónicas, notas y entrevistas}

BOY (seud. de Antonio SOTO). La barraca de Bertani. En: El Plata, Montevideo. [Recorte sin data hallado en el archivo particular de Sra. Perla de Oriente Bertani].

CASTRO, Manuel de. Desaparece el viejo Café Británico. En: La Mañana, Montevideo, 13 marzo 1955, Suplemento Femenino, p. 20.

CASTRO, Manuel de. El editor Orsini Bertani y la cultura uruguaya. En: La Mañana, Montevideo, 12 jun. 1955, Suplemento Femenino, p.20. (Serie de artículos "Cronicones montevideanos").

CASTRO, Manuel de. Los avatares de Orsini Bertani. En: La Mañana, Montevideo, 4 feb. 1956, Suplemento Femenino, p. 20.

CASTRO, Manuel de. Claudio García, editor y librero. En: $L a$ Mañana, Montevideo, 30 sept. 1956, Suplemento Femenino, p. 3.

CASTRO, Manuel de. Evocaciones de la ciudad. Rubén Darío en Montevideo: Consagración de Julio Herrera y Reissig y Delmira Agustini. En: Mundo Uruguayo, Montevideo, 22 sept. 1960, s/p.

DE GREGORIO, Juan (seud.: David). La librería Florencio Sánchez. En: Mundo Uruguayo, Montevideo, 13 dic. 1945, p. 8-9.

DURAND, Elizabeth. Fuimos al reencuentro de Orsini Bertani. En: El Día, Montevideo, 17 agosto 1980, Suplemento Dominical.

FERREIRO, Alfredo Mario. El 'Polo Bamba' se vino desde la calle Colonia hasta la Plaza Independencia para mostrarle a Montevideo la sin par calidad de su clientela. En: La Razón, Montevideo, 23 enero 1945, Página "Martes Literario".

LASPLACES, Alberto. Los cafés literarios: Recuerdos del 'Polo Bamba'. En: El Día, Montevideo, n. 466, 21 dic, 1941, Suplemento Dominical.

MORA GUARNIDO, José. Panorámica y postrimerías del Tupí-Nambá. En: Montevideo Altillos Cafés Literatura, Pablo Rocca (antología y prólogo). Montevideo: Arca, 1992. p. 101-124. [Originalmente en Revista Nacional, Montevideo, n. 202, oct.-dic. 1959].
MORATORIO, Orosmán. Tiempos de la vieja calle Sarandí: La silueta de Roberto. En: La Mañana, Montevideo, 2 jun. 1946.

MÉRICA, Ramón. Vera historia del visionario editor que marcó a la cultura del Uruguay... En: El País, Montevideo, 24 sept. 1995. p. 10-11. [Entrevista a Perla Bertani].

RUFFINELLI, Jorge. [1971]. Alberto Zum Felde: el magisterio de la conciencia crítica. En: Palabras en orden. México: Universidad Veracruzana, 1985. p. 9-35. [Entrevista].

SILVA VALDÉS, Fernán. Autobiografía [Tercera parte]. En: Revista Nacional, Montevideo, n. 200, p. 206-216, abr.-jun. 1959.

VISCA, Arturo Sergio. Conversando con Zum Felde. Montevideo: Biblioteca Nacional, Departamento de Investigaciones/Reportajes Culturales, 1, 1969. [Entrevista].

ZUM FELDE, Alberto. Testimonio sobre Orsini Bertani. Original mecanografiado, dos folios, con correcciones manuscritas. [Original en poder de la Sra. Perla Bertani. Consultado en 2004].

\section{Miscelánea sobre Orsini Bertani}

(entradas por orden cronológico)

\section{Sobre su muerte}

SIN FIRMA. El señor Orsini M. Bertani. Falleció ayer. En: El Día, Montevideo, 17 marzo 1939.

SIN FIRMA. Orsini Bertani. Su fallecimiento. En: El Plata, Montevideo, 17 marzo 1939.

SIN FIRMA. Murió Orsini Bertani, el noble y generoso editor. En: El Pueblo, Montevideo, 18 marzo 1939.

SIN FIRMA. Con Orsini Bertani desaparece un valiente luchador demócrata. En: Crítica, Buenos Aires, 18 marzo 1939.

SIN FIRMA. Ayer fueron inhumados los restos del señor Orsini M. Bertani. En: El Día, Montevideo, 18 marzo 1939.

SIN FIRMA. Breve reseña biográfica de Orsini Bertani. En: España Democrática, Montevideo, 31 marzo 1939, p. 4, col. 1.

GARCÍA PUERTAS, Manuel. Una extraordinaria demostración de dolor fue el sepelio. En: España Democrática, Montevideo, 31 marzo 1939, p. 4, cols. 2-4.

FERNÁNDEZ RÍOS, Ovidio. Discurso del vice-presidente del Círculo ‘El Progreso' don Ovidio Fernández Ríos. En: España Democrática, Montevideo, 31 marzo 1939, p. 4, cols. 4-5.

\section{Otros homenajes póstumos}

SIN FIRMA. A un año de la muerte de Orsini M. Bertani. En: Progreso, Montevideo, n. 5, marzo 1940, p. 1.

SIN FIRMA. El Progreso y los italianos. En: Diario Popular, Montevideo, 19 jun. 1943, p. 3.

FERNÁNDEZ RÍOS, Ovidio. Mañana en El Ateneo se recordará a Orsini Bertani. Luchó por la libertad y los derechos sociales como hombre y como editor. En: El Día, Montevideo, 17 sept. 1959.

SIN FIRMA. El editor Orsini Bertani será evocado en El Ateneo. En: El País, Montevideo, 17 sept. 1959.

SIN FIRMA. Orsini M. Bertani evocado por el C[entro] de Artes y Letras ‘Á[ngel] Falco’. En: El Día, Montevideo, 20 sept. 1959.

Recebido: 13/01/2018

Aprovado: 20/01/2018

Contato:

Pablo Rocca <pabloroccapesce@gmail.com> 